\title{
Intraoral Swallow Pressure Profiles: General Features and Aids to Categorization
}

\author{
Vincent Casey $^{1}$ (D) Alison Perry ${ }^{2} \cdot$ Richard Conway $^{3}$
}

Received: 13 August 2021 / Accepted: 11 January 2022 / Published online: 4 February 2022

(c) The Author(s) 2022

\begin{abstract}
Purpose The primary goal of this study was to establish a normative data set representing intraoral time series swallow pressure profiles for healthy adults using a novel wearable intraoral pressure sensing system, OroPress, developed to help with dysphagia (swallow disorder) clinical screening.

Methods Swallow intraoral pressure-time profiles for 35 healthy adults (17 male, 18 female) swallowing water $\left(3 \times 5 \mathrm{~cm}^{3}\right.$; $\left.3 \times 10 \mathrm{~cm}^{3}\right)$ and custard $\left(3 \times 5 \mathrm{~cm}^{3}\right)$ boluses $(N=9 \times 35=315)$ were recorded using OroPress.

Results General swallow profile traits are identified to characterise an effective, efficient swallow. A profile-specific swallow envelope function is devised which in combination with profile metrics, provides a simple means of categorizing swallows as effective or impaired.

Conclusion The swallow profile data trace with superimposed and colour coded peaks, envelope function and related swallow metrics provides a simple human readable graphic to aid the real-time instrumented identification of subjects warranting more in-depth clinical assessment. It may also prove useful in the selection of training set profiles for machine learning and other analysis tools which could improve the discriminatory capabilities of intraoral pressure measurement in dysphagia diagnostics.
\end{abstract}

Keywords Swallow pressure $\cdot$ Envelope function $\cdot$ Dysphagia screening

$\begin{array}{ll}\text { Abbreviations } \\ \text { EMG } & \text { Electromyography } \\ \text { PCA } & \text { Principle component analysis } \\ \text { LDA } & \text { Linear discriminant analysis } \\ \text { HP } & \text { Hydrostatic pressure } \\ \text { IP } & \text { Interfacial pressure } \\ \text { BIPT } & \text { Biomedical interface pressure transducer } \\ \text { PEMAX } & \text { Maximum pressure excursion } \\ \text { Pmax } & \text { Maximum pressure }\end{array}$

Clinical data collection was undertaken by Carmel Ni Chualain, Catraoine Hickey, Joanne McCormack as part of their Honours MSc Degree in Speech and Language Therapy.

Vincent Casey

vincent.casey@ul.ie

1 Department of Physics, University of Limerick, Limerick, Ireland

2 School of Allied Health, University of Limerick, Limerick, Ireland

3 Department of Electronic and Computer Engineering, University of Limerick, Limerick, Ireland
Pmin Minimum pressure
PEXS Positive excess pressure
NEXS Negative excess pressure
PWHM Pulse width at half maximum
Pt Pressure time product

\section{Introduction}

Dysphagia has been extensively investigated using a variety of techniques including radiography, ultrasound, electromyography (EMG), videofluoroscopy [1] and endoscopy together with direct observations of individuals with dysphagia [2]. Intraoral swallow pressure-time profiles offer a relatively non-invasive means of interrogating the swallow process and so have been used in many studies designed to improve our general understanding of normal and impaired swallowing [3-5]. Researchers tend to use pressure peak amplitude [6-8] and pressure rise/fall times [9] as dependent variables. While these variables have an immediate intuitive appeal, their discriminatory capabilities are limited given the large dynamic ranges encountered across different studies 
but also within a given study and even within individual swallows. The variety and complexity of swallow pressure profiles reflects, to some extent, the complexity of the swallow process [10] but pressure measurement artifacts and lack of control of independent variables is also likely to add to the variety of profiles captured [11]. Nevertheless, it has been possible to identify general features in profiles for healthy volunteers $[12,13]$ which provides some encouragement in the search for a universal profile which could be used as a reference to identify subjects with impaired swallow function.

Statistical techniques such as principal component analysis (PCA), linear discriminant analysis (LDA) and machine learning may provide more robust metrics with improved discrimination [14]. However, a 'normal' swallow profile set, the so-called training set, obtained from individuals with unimpaired tongue function or swallow function is required [15]. Selecting a 'normal' population sample is difficult since participants may not be aware of, or manifest with clear symptoms of dysphagia. In addition, tongue function impairment is understood to increase with age [16], affecting, according to some studies, more than $40 \%$ of adults over 50 [17]. Therefore, a sample drawn from a healthy adult population is unlikely to guarantee a robust training set unless rigorous invasive clinical screening is used to identify healthy participants.

There is a need therefore, both from a clinical and an analysis perspective, for a simple objective tool or instrument which could be used to classify human oral swallow pressure profiles into normal or impaired swallow categories in real time. The OroPress system [18] was developed in an attempt to meet this need.

\section{Background}

Tongue pressure generation and articulation within the oral cavity is a critical element of normal speech and swallow function [12] and involves the volitional and reflexive activities of more than 30 nerves and muscles [10]. Two distinct kinds of pressure may arise within the oral cavity during liquid bolus swallowing. The bolus hydrostatic pressure $H P$ may be changed by applying a force which tends to change the volume of the bolus compartment created by the tongue. According to Pascal's principle [19], and assuming a low viscosity liquid bolus, the pressure generated within the bolus will be uniform or constant throughout the liquid contained within the compartment and may be positive or negative relative to ambient pressure [13, 20]. In forming a container for the bolus the tongue also forms a seal around the bolus through tongue contact with other tissue structures. Provided shear forces are decoupled leaving only normal forces, the seal pressure may be measured as a biomedical interface pressure $I P$ [21]. The effectiveness of swallow function is expected to depend on the mechanical integrity and effectiveness of compartment and seal formation [22], and swallow efficiency similarly might be expected to depend on the bolus transport efficiency of the tongue [23]. In this context, an effective, unimpaired swallow can be defined as one which forms and dispatches the bolus to the stomach with minimum mechanical effort (force/work/ energy) and in minimum time. Consequently, direct intraoral tongue pressure measurement during swallowing can provide an objective means of monitoring the mechanical effectiveness of a swallow, not only during the oral phase of swallowing but for subsequent phases also since this effort continues until the swallow is complete [24]. Hence the large number of studies focusing on pressure measurement during swallowing in the dysphagia literature.

A range of commercial instruments and approaches exist [6] for swallow and tongue function measurement such as the Kay Swallowing Workstation [25], the Iowa Oral Performance Instrument [26] and the Madison Oral Strengthening Therapeutic [27]. In general these systems rely on deformation by the tongue of an elastic element such as a gas filled elastomer bulb which is linked via a tube to a remote pressure sensor which in turn measures the changes in pressure in the gas due to deformation of the bulb. The bulb deformation and consequent gas pressure change is used as an indicator of the strength and movement of the tongue during swallowing. However, while pressure sensors may be used in these devices, the probes used do not measure intraoral pressures. To measure such pressures, the pressure sensors need to be located within the oral cavity or coupled directly to it using an open ended tube.

It is now possible to modify microelectromechanical sensors (MEMS) pressure sensor modules to measure intraoral pressures directly using probes [28] which are relatively non-intrusive and which provide absolute measures of pressure, i.e., can measure both positive and negative $H P$ values relative to ambient atmospheric pressure but which will also respond to biomedical $I P$ values $[11,29]$. The sensitivity of these sensors to both $H P$ and $I P$ is likely to further add to the complexity of the swallow pressure profile. However, such complexity is probably a realistic reflection of the complexity of the actual processes involved in swallowing. Such profiles may therefore contain detail sensitive to bolus related properties such as temperature, consistency/viscosity [30], voluntary or on-command swallowing [9] as well as subject attributes such as gender [31], age and habituation. The major challenge is to identify the general or, if possible, universal features of such profiles to allow the extraction of clinically meaningful metrics indicative of the effectiveness and efficiency of a swallow.

Some very general intraoral swallow pressure profile features may be identified from the dysphagia literature. First, 
a swallow pressure profile is continuous and may comprise multiple peaks but generally has one dominant peak most simply characterised by its maximum pressure value. Second, the duration of such profiles depends on bolus consistency and volume but is typically less than half a second for saliva $[6,32]$ and somewhat longer but less than one second for water and custard. Third, the area between the pressure profile and the time axis serves as an index of the overall 'effort' or energy expended in swallowing $[12,33]$ the bolus. Clearly there is an interdependence between these features. However, they provide a basis for a simple definition of an effective swallow, in the context of intraoral pressure-time profiles, as one in which the bolus is transported from the mouth to the stomach in the minimum of time and with the minimum of effort. Furthermore, while the discriminatory value of the maximum pressure may be limited, its uniqueness within each profile identifies it as an ideal alignment feature in order to compare swallow profiles. These general profile features and the uniqueness of the profile maximum value are used to guide the analysis of swallow profile data here.

\section{Experimental Details}

The OroPress system [18] was used to directly monitor and record intraoral pressure and temperature during liquid swallowing and isometric strength and endurance tasks. This system uses a modified MEMS pressure sensor (biomedical interface pressure transducer (BIPT) [29]) capable of responding to both bolus $H P$ and tissue $I P$, connected to a wearable wireless module. The wireless module transmits real time data to a remote notebook computer which displays and records the data as well as participant and clinician details. The pressure data is absolute, i.e., referenced to vacuum. A baseline may be established by ensuring that the participant's mouth is open at commencement of the testing sequence, i.e., zeroed while exposed to ambient atmospheric pressure.

The measurement protocol, participant recruitment and screening details and results for isometric strength and endurance tests carried out as part of this study have been reported previously [18]. A preliminary attempt to use PCA on the swallow data has also been reported [34]. A total of $N=35$ normal healthy adults were purposefully recruited (17 males and 18 females) ranging in age from 18 to 70 years (average age 36) and screened as admissible to the study. Each participant was seated facing a wall $2.4 \mathrm{~m}$ distant on which was placed a viewing disc target at subject eye-level. The BIPT probe was adhered to the participant's alveolar ridge using a Poligrip ComfiSeal Strip ${ }^{\circledR}$. Each participant first practised oro-lingual isometric tongue 'pushing' pressure tasks to become habituated to the probe. Once they indicated that they were comfortable, recorded tests commenced. Participants were instructed to gaze at the disc during data collection to ensure that their head was stabilized in a neutral position, i.e., neither tilted up nor down. Isometric (strength and endurance) and swallow tasks were performed in counterbalanced order.

Swallow data was obtained for randomized sequential sets of three boluses for each participant: $3 \times 5 \mathrm{~cm}^{3}$ water; $3 \times 10 \mathrm{~cm}^{3}$ water and $3 \times 5 \mathrm{~cm}^{3}$ custard. The bolus was drawn from fridge stored stock and delivered to the oral cavity using a syringe. The participant was instructed to hold the bolus in their mouth briefly before swallowing on command. Once completed, the participant test data was saved to individual participant Microsoft (MS) Excel® spreadsheets. The swallow segment of the participant data set thereby comprised a total of 9 swallows. A total of $9 \times 35=315$ swallow pressure profiles were recorded in this clinical study.

Individual swallow data sets for a single participant were extracted into sequential columns of an MS Excel spreadsheet. Once all nine swallows were extracted for a particular participant, the entire procedure was repeated for each other participant data file. Swallow durations varied so column lengths in the spreadsheet varied accordingly. Once all participant swallows were extracted (swallow vectors), the spreadsheet containing the swallow column vectors, see https://osf.io/7c2va/ Resource 1, was imported to MATLAB (Mathworks, Natick, Massachusetts, USA) as a matrix of swallow vectors. The matrix row size (number of rows), corresponded to the longest swallow vector imported which was 700 and so the $2 \mathrm{D}$ swallow matrix dimension was $315 \times 700$. Custom MATLAB scripts, described below, were developed to extract swallow parameters, e.g. maximum positive and negative excursion pressures, as well as to carry out general numerical processing of the matrix of swallow vectors.

\section{Results and Analysis}

An example of a participant extracted swallow set profile recorded in this study is shown in Fig. 1 where the zero baseline corresponds to ambient atmospheric pressure. The data rate is 100 readings (pressure and temperature) per second, i.e., pressure and temperature are recorded every $0.01 \mathrm{~s}$. The temperature data is used to compensate the pressure data for temperature induced changes. However, it is also useful as an indicator of bolus presence around the BIPT probe provided there is a temperature contrast between bolus and body temperatures. For instance, the temperature trace shown displays localised drops in temperature once a bolus is introduced (fridge stock bolus is at a temperature lower than body temperature). A saliva swallow, on the other hand, does not, in general, display an accompanying temperature drop. However, a bolus 


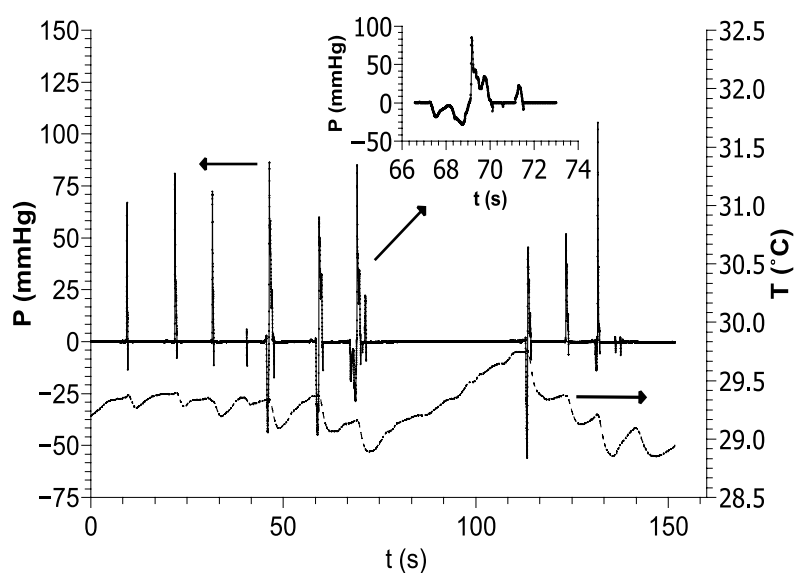

Fig. 1 Pressure and temperature profile for 3 sets of 3 swallows in the order: $3 \times 5 \mathrm{~cm}^{3}$ water; $3 \times 5 \mathrm{~cm}^{3}$ custard; $3 \times 10 \mathrm{~cm}^{3}$ water. Insert shows a time-axis expanded single swallow

clearance swallow of residual bolus will have an associated temperature drop, so will be distinguishable from a saliva swallow thereby aiding swallow selection and isolation. Swallows extracted in this way were validated by reference to swallow times/locations noted by the clinicians.

The inset to Fig. 1 shows an expanded time-base view of a single swallow pressure profile. The pressure fluctuates above and below the baseline, i.e. comprises positive and negative pressure excursions relative to atmospheric pressure. It has a clear start defined by the departure of the pressure from baseline and a clear completion point defined by a return to baseline. There is evidence of a short clearance swallow just before $72 \mathrm{~s}$ since the temperature continues to drop across the entire swallow profile sequence. For this particular swallow the maximum pressure excursion Pmax, from baseline, is positive and close to $90 \mathrm{mmHg},[1 \mathrm{kPa} \simeq 7.50 \mathrm{mmHg}]$. MATLAB 'max' and 'min' functions were applied to the swallow matrix to extract the maximum positive, Pmax, and maximum negative/minimum, Pmin, pressure excursion values from baseline for each swallow. Out of 315 swallows recorded, the maximum pressure excursion, Pmax, was positive for 287(91\%) swallows, i.e., $P \max >|P \min |$, and negative for $28(9 \%)$ swallows of which 14 were attributable to just 3 participants. The Pmax mean and median values were $154 \mathrm{mmHg}$ and $118 \mathrm{mmHg}$ respectively while the corresponding values for Pmin were $-41 \mathrm{mmHg}$ and $-19 \mathrm{mmHg}$ respectively. In all, 95\% of Pmax values are less than or equal to $380 \mathrm{mmHg}$ while the corresponding value for Pmin values is $-135 \mathrm{mmHg}$. Therefore, while the pressure range of peaks encountered for all swallows spans just over $1300 \mathrm{mmHg}$ (maximum of $630 \mathrm{mmHg}$ to minimum of $-690 \mathrm{mmHg}$ ), $95 \%$ fall within an approximately $500 \mathrm{mmHg}$ dynamic range.

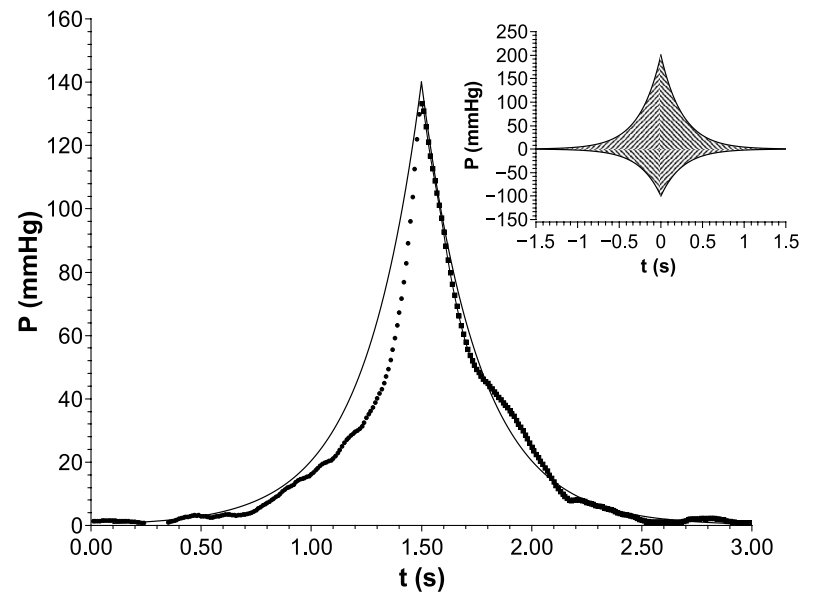

Fig. 2 Averaged pressure data for all aligned swallow profiles at corresponding times within the $3 \mathrm{~s}$ profile interval. The solid curves correspond to exponential 'best-fit' functions with amplitude $140 \mathrm{mmHg}$ and time constant $0.26 \mathrm{~s}$. The insert shows composite envelope function plots centered on Pmax with different positive $(200 \mathrm{mmHg})$ and negative $(-100 \mathrm{mmHg})$ amplitudes and fixed time constant $\tau=0.26 \mathrm{~s}$

The maximum pressure excursion value Pmax, as noted above, provides a unique marker within each swallow profile. A MATLAB script was used to align all profile vectors on their Pmax value (Pmax or Pmin depending upon which had the greater absolute value), see https://osf.io/n9gw8/ Resource 2 . Swallow vectors were truncated $\pm 1.5 \mathrm{~s}$ either side of the maximum pressure excursion as a $3 \mathrm{~s}$ sample window was judged to be sufficient to cater for both normal and 'impaired' swallow profiles. Where a profile was less than $3 \mathrm{~s}$ duration, it was padded with zero values in the aligned swallow vector matrix. Such time shifting and temporal truncation/padding of pressure profiles greatly facilitates profile comparison, (see data file density plot).

Terminology used in amplitude envelope analysis of sound events [35] is useful in describing these pressure profiles. The swallow effort starts to build on the leading edge or 'attack' side of maximum and builds up slowly about $1 \mathrm{~s}$ before maximum for a large number of swallows, increasing more rapidly within $0.5 \mathrm{~s}$ of maximum. On the 'release' or falling edge side of maximum the effort drops rapidly for the first $0.5 \mathrm{~s}$ after maximum returning slowly to baseline thereafter. Averaging swallow pressures for all aligned profiles at each time point provides a generalised profile shape for these liquid swallows, Fig. 2, irrespective of bolus type. Regression analysis of the data $0.75 \mathrm{~s}$ either side of maximum gave a reasonable fit $\left(R^{2}>0.97\right)$ to exponential attack and decay functions with amplitude $140 \mathrm{mmHg}$ and time constant $\tau=0.26$ s, i.e. $P=140 \exp \pm((t-1.50) / \tau)$, Fig. 3. Based on this, a swallow envelope function was created in MATLAB composed of a set of exponential functions, parameterised using two amplitudes (the Pmax and Pmin values for each profile) and a single time constant $\tau=0.26 \mathrm{~s}$, and 


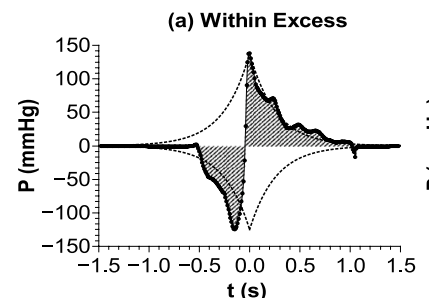

(c) Negative Excess
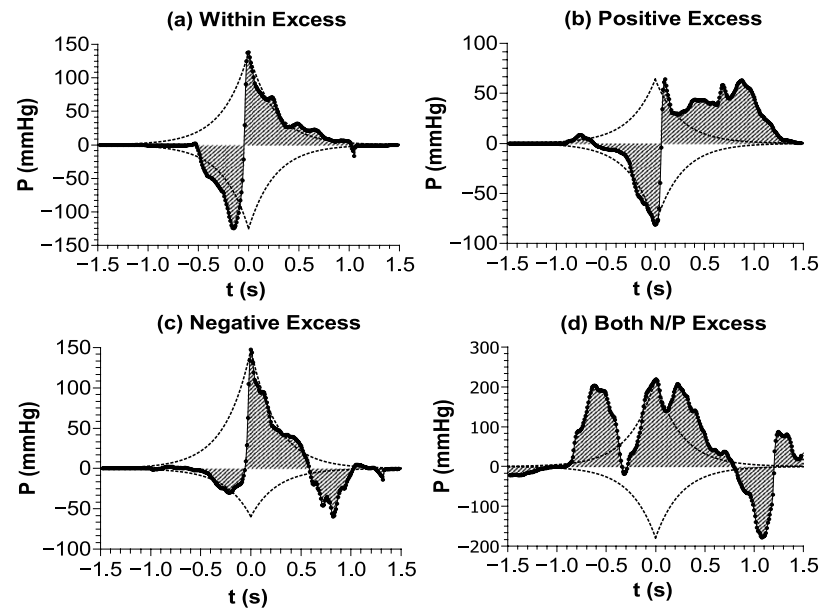

Fig. 3 Examples of swallow profiles: a within the envelope excess thresholds; b exceeded the positive envelope excess threshold only; c exceeded the negative envelope excess threshold only; $\mathbf{d}$ exceeded both positive and negative envelope excess thresholds

formed into a composite swallow envelope function with, where appropriate, positive and negative attack and release components, see insert to Fig. 2. Thus the profile envelope functions were normalised to individual profile maximum/ minimum values but a single time constant, $\tau=0.26 \mathrm{~s}$ was used for all profiles.

Using the customized envelope functions for each profile, as described above, it is possible to devise metrics to indicate the degree to which a given profile conforms to, or deviates from, the envelope profile. Actual swallow profile pressure values were compared with envelope values for all time-series data points within the $3 s$ profile window, i.e., $\pm 1.5 \mathrm{~s}$ of Pmax. Pressure values $P_{i}$ greater than the positive envelope values $P E_{i}$ or less than the negative envelope values $N E_{i}$ were individually summed in order to provide numerical estimates of the $P t$ area which was outside the positive envelope bounds, PEXS, and negative envelope bounds, $N E X S$, for each swallow profile, i.e., $P E X S$ or $N E X S=\sum_{i=1}^{300}\left(\left|P_{i}\right|-\left|P E_{i}\right|\right) \times \Delta t$.

Cumulative frequency analysis https://osf.io/bf8sw/ Resource 3 of the excess values showed that small excess values dominate with $91 \%$ of all PEXS values (all participants, all swallows) being less than or equal to $50 \mathrm{mmHg}$. The corresponding value for $N E X S$ is approximately $14 \mathrm{mmHg}$ s. These values were used as threshold levels to categorize swallow profiles according to whether they were within thresholds or, if outside, whether they exceeded positive, negative or both thresholds. Sample profiles from each of these categories are shown in Fig. 3 along with their corresponding envelope functions. Correlation analysis of all swallow excesses and total excesses for each participant and age yielded positive and weak negative coefficients all less than 0.4, see https://osf.io/hp4bg/ Resource 4. Even within
Table 1 Summary data for the 17 participants with swallow profiles with threshold excesses

\begin{tabular}{llllll}
\hline Both & Positive only & Negative only & Total & Gender & Age \\
\hline $7(1)$ & $7(6)$ & $7(1)$ & 8 & F & 26 \\
& $19(1)$ & & 1 & F & 27 \\
& $6(4)$ & & 4 & F & 27 \\
& & $26(1)$ & 1 & F & 31 \\
& $20(1)$ & & 1 & F & 34 \\
$13(3)$ & $13(3)$ & $13(3)$ & 9 & F & 42 \\
$25(1)$ & $25(2)$ & & 3 & F & 57 \\
& $9(2)$ & $9(1)$ & 3 & F & 70 \\
& & $16(4)$ & 4 & M & 19 \\
& & $24(1)$ & 1 & M & 19 \\
& $14(1)$ & $14(2)$ & 3 & M & 21 \\
& & $11(1)$ & 1 & M & 28 \\
& & $1(1)$ & 1 & M & 34 \\
& & $34(1)$ & 1 & M & 41 \\
& & $31(2)$ & 2 & M & 43 \\
& & $4(3)$ & 3 & M & 45 \\
& & & 4 & M & 62 \\
& $35(4)$ & 21 & 50 & & \\
\hline
\end{tabular}

The table gives the participant number and number of swallows (in brackets) which exceed both excess thresholds, positive threshold only or negative threshold only

gender subgroups, correlations between excess pressures and age were weak. In all 24 (7.6\%) swallow profiles were found to exceed the positive excess threshold only, Table 1. A further $21(6.7 \%)$ exceeded the negative excess threshold only and $5(1.6 \%)$ profiles exceeded both the positive and negative excess thresholds giving a total of $50(15.8 \%)$ profiles which exceeded one or both thresholds. Table 1 provides details for participants with threshold excesses grouped according to gender and in order of increasing age within each gender subgroup. While 17 of the 35 participants had one or more swallows which exceeded the thresholds, one participant (PN13) had all 9 swallows, $P N 07$ had 7, while participants $P N 06, P N 16$ and $P N 35$ each had 4 swallows in excess of threshold thereby accounting for more than half those identified as having profiles with significant portions outside the swallow envelope, Table 1 . Summary data such as that shown in the table could serve to identify participants warranting referral to a clinical specialist since they appear to be using anonymously large effort in swallowing.

While the envelope function provides a simple graphical aid to profile interpretation and yields metrics relating to the degree of profile conformation to the envelope function, metrics commonly used in standard signals analysis can also prove useful in the placement of a particular swallow on a swallow effectiveness scale ranging from effective to impaired. The predominance of a single maximum pressure excursion peak leads naturally to a consideration of the pulse 
width at half maximum $P W H M$ as a measure of swallow duration. The $P W H M$ values for all swallows were computed in MATLAB by finding the index for Pmax and then finding the indices either side of Pmax where the pressure first drops to half $P \max$, i.e., $P \max / 2$. The $P W H M$ and pulse rise and fall times (time for pressure to increase from half maximum to maximum on the attack side or decrease from maximum to half maximum on the release side respectively) were calculated directly from these indices. A histogram of $P W H M$ values is shown in Fig. 4 with an inset showing a box-plot for $P W H M$ and the rise and fall times. A lognormal distribution function gives a reasonable fit to the PWHM data which ranged from 0.02 to $1.20 \mathrm{~s}$. The boxplots show that $90 \%$ of $P W H M$ values fall in the range $0.20-0.70 \mathrm{~s}$ and that the rise and fall times are similar to each other and close to $0.4 \mathrm{~s}$ reflecting a high degree of symmetry in the dominant pressure pulse(s) centered on Pmax.

As is clear from Fig. 1 multiple subsidiary peaks in addition to the maximum pressure excursion peak may arise in these intraoral pressure profiles. A very useful peak finder function [36] developed for MATLAB ${ }^{\circledR}$ may be used to count peaks in time-series data. Using this function, positive and negative peaks were counted based on their magnitude relative to $P \max$ (because of the dominance of positive pressure peaks) for a given swallow profile. Positive peaks were counted provided their maximum value was above a threshold of Pmax/5 and that they were at least Pmax/10 greater than surrounding data. Similarly, negative peaks were counted provided the peak's absolute value was greater than $P \max / 5$ and that it was at least $P \max / 10$ greater than surrounding data, i.e. more negative. Positive and negative peaks were counted separately and added to provide a total peak count for the time interval $\pm 1.5 \mathrm{~s}$ of $P \max$, i.e., a $3 \mathrm{~s}$ interval. A histogram of total peak count is shown in Fig. 5 along with an inset which shows box plots for individual peak counts and total peak count. Swallow profiles had a dominant (single peak) for 58 swallows (18\%) whereas 116 swallows were double peaked (37\%). The maximum peak counts per swallow were 4 for profiles with negative peaks, 6 for profiles with positive peaks and the maximum total peak count per swallow was 8 , i.e., comprising both positive and negative peaks. For total peak count, $95 \%$ of swallow profiles had 5 or less peaks, with $75 \%$ having 3 or less peaks. The median total peak count was 2 . A log-normal distribution function provides a reasonable fit $(P=0.17$ at the 0.05 level, Shapiro-Wilk for transformed data).

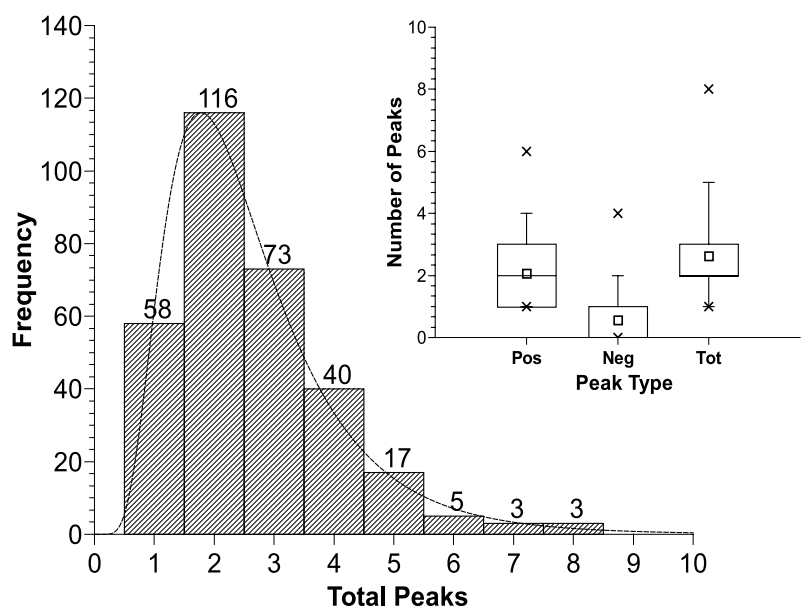

Fig. 5 Histogram of total peak count per swallow. The inset shows box plots for positive, negative and total peak counts. (Markers as for Fig. 4)

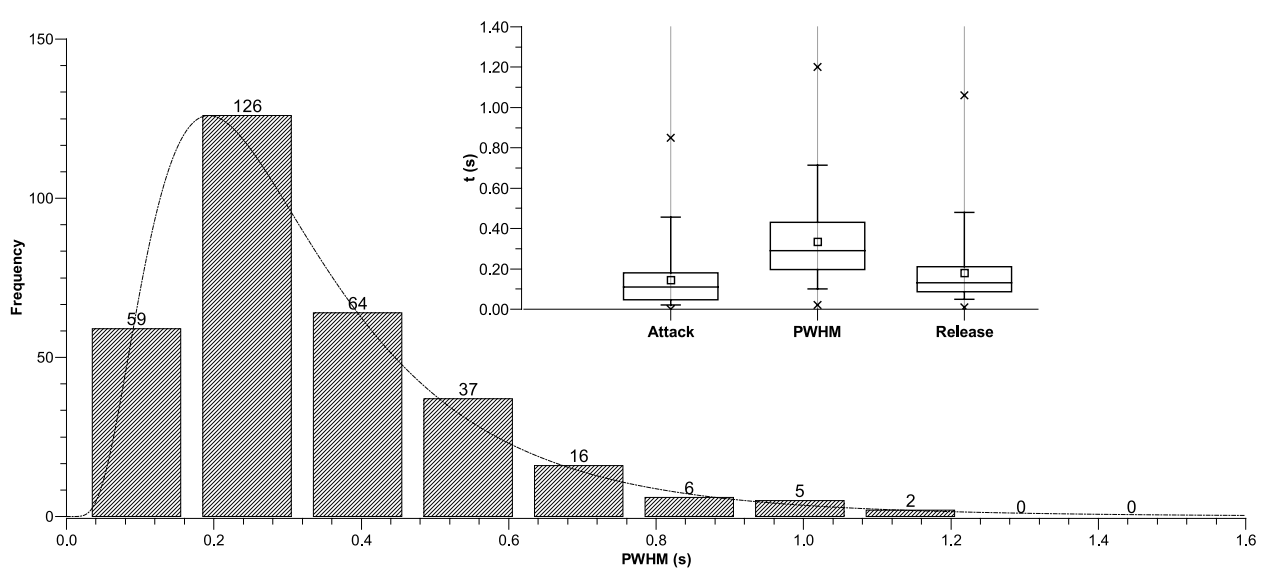

Fig. 4 Box plots of Pulse Width at Half Maximum (PWHM) for the maximum magnitude peak within each swallow profile. The 'Attack' plot refers to the time taken to rise from half maximum to maximum on the commencement portion of the profile whereas the 'Release' plot refers to completion portion of the profile, i.e., time take to fall from maximum to the first instance of half maximum. (Whiskers correspond to the 5 and $95 \%$ limits, the large rectangle gives the interquartile range, the line across the large rectangle is the median value and the small square is the mean value) 
The pressure-time product was summed in order to estimate the profile area, i.e., Pt product, see https://osf.io/ bf8sw/ Resource 3, as an "effort index", for both the positive and negative pressure profile elements for each profile $1.5 \mathrm{~s}$ either side of Pmax for each swallow, i.e., $\left(\sum_{i=1}^{300}|P| \times \Delta t\right)$, where $\Delta t=0.01 \mathrm{~s}$ is the sampling interval. Total effort, gauged via the $P t$ product, is typically less than $230 \mathrm{mmHg} \mathrm{s}$ (within 95 percentile). More than $75 \%$ of swallows had a total pressure-time effort value below $100 \mathrm{mmHg} / \mathrm{s}$ with $5 \%$ of swallows having values less than $20 \mathrm{mmHg}$ s. All swallows were clinically assessed as being effective, yet the effort involved (without differentiating between bolus volume or type since these are similar on an order of magnitude basis), spans two orders of magnitude. The wide range of effort involved in swallowing and the dominance of positive pressure-time products is possibly due to the tongue pressing on the sensor to provide a sealing function rather than a propulsive effort (probe location close to or at the alveolar ridge).

\section{Categorization Aid}

In order to demonstrate how an OroPress intraoral pressure profile might be used as a real time screening tool to assess the effectiveness of human swallowing, a script was written in Python, see https://osf.io/68a5c Resource 5 which takes a swallow profile, truncates it \pm 1.5 s of Pmax, generates the corresponding envelope functions and "effectiveness" parameters. It then plots and displays the profile and envelope functions, annotated with parameters, peak markers and highlighted envelope excess areas, as an easily interpreted single page graphic, Fig. 6. If implemented on the OroPress data acquisition device (Laptop/Hand-held device), this graphic provides a simple real time output which may prove useful in initial clinical screening for dysphagia. Clearly,

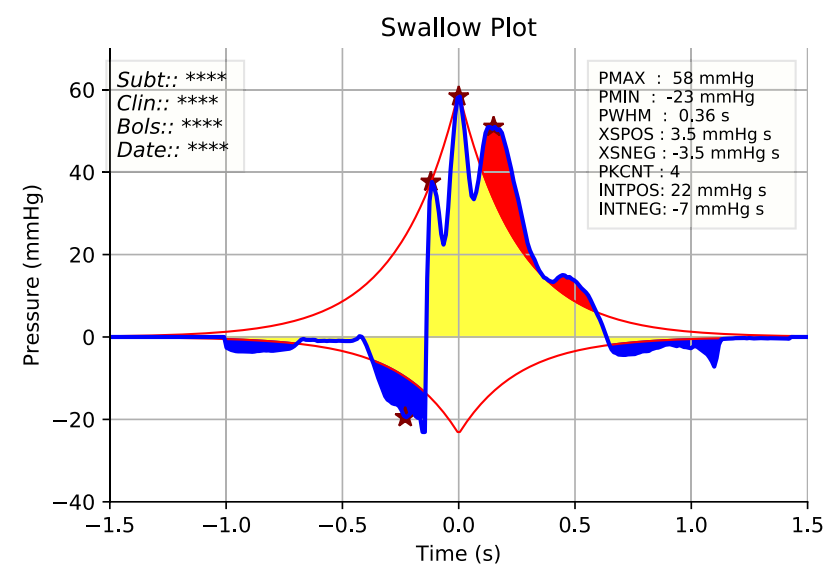

Fig. 6 Python generated plot of swallow profile, envelope functions and parameters with marked positive and negative peaks and highlighted envelope excess areas the tool needs to be tested and validated through use on subjects with swallow disorder. Application to OroPress data recorded on head and neck cancer patients who have undergone radiotherapy treatment will be presented in a subsequent paper.

\section{Discussion}

Tongue movement is varied and active throughout the entire swallowing process [24] involving volitional and reflexive activities of more than 30 nerves and muscles [10]. A pressure sensing device placed directly in the oral cavity will provide swallow profile data for the entire swallow, not just the oral phase, particularly if the device is sensitive to both $H P$ and $I P$. The partly reflexive, partly voluntary nature of the tongue motor activity during swallowing is likely to contribute to the large variety of pressure profiles observed and makes it difficult to define a normal or universal profile in contrast to the situation which pertains to ECG heart monitoring. Nevertheless, as has been demonstrated, there are distinct patterns to the pressure signals and consistent patterns are present in multiple sensor signals from the same subject and across subjects [12].

Two easily extracted but interdependent features of intraoral swallow pressure profiles were identified: profile duration characterised by the pulse width half maximum, $P W H M$ and the swallow effort characterised by the profile pressure-time product, $P t$. These could be used as a basis for a simple classification scheme for liquid bolus swallow effectiveness. One such scheme could define an effective swallow as one which would transport the bolus from the mouth to the stomach in the minimum of time, i.e. $P W H M<0.3 \mathrm{~s}$, and with the minimum of effort, $P t<150 \mathrm{mmHg}$.

The value of Pmax is less useful as a characterization parameter since it can relate to a combination of $H P$ and $I P$. However, since the maximum pressure excursion is single valued and therefore unique within each profile, it is useful as an alignment feature for all profiles in order to allow easy profile comparison. A profile window of $\pm 1.5 \mathrm{~s}$ either side of Pmax may be sufficient to capture both effective and ineffective swallows.

A superposition of all Pmax aligned swallow profiles revealed relatively high data density within a set of envelop functions characterised by two amplitudes (one positive, the other zero or negative) and a single time constant centered on Pmax. Swallows could then be classified into groups based on the degree to which they exceeded or strayed outside the envelope functions. Such envelope functions may prove useful as aids to the identification of participants/ patients requiring further clinical assessment.

It is easy to combine graphically rendered swallow profiles with numeric metrics such as $P W H M$, peak count, Pmax 
and Pmin. Numerical processing also facilitates assessment of swallow effort from pressure profiles through the pressure-time Pt product and through PEXS and NEXS. A display of the profile with its envelope function augmented with parameters such as those mentioned above, if available in real time, i.e., contemporaneous with swallow testing, could add significantly to our understanding of swallow pressure profiles and if combined with rigorous clinical evaluation/ asssessment, could also provide an evidence base supporting the use of the tool and this approach in clinical preassessment for dysphagia, e.g., Fig. 6

Because the analysis of profiles reported here was done subsequent to swallow testing, it was not possible to do follow up clinical assessments of participants with multiple swallows having envelope excesses outside the thresholds. This represents a major limitation in the study since it was not possible to identify whether such anomalous profiles and associated parameters are attributable to actual swallow difficulties, i.e. dysphagia, probe invasiveness or other causes. Related to this limitation is the general difficulty with selecting a sample participant population which has unimpaired swallow function. Currently, there is no reliable way of doing this without resort to videofluoroscopic or other relatively invasive screening.

In an attempt to overcome these limitations a further study has been approved and completed where the participants are head and neck cancer patients who have had radiotherapy treatment. OroPress has been used in parallel with videofluoroscopy on a small group of such patients. Analysis is on-going and results will be reported

Author Contributions VC and RC designed and developed the OroPress tool in consultation with AP. AP supervised the clinical use of OroPress on healthy volunteers by MSc speech and language therapy students. VC developed the code to support the research, and carried out the post processing of raw pressure-time series swallow data and the analysis and presentation of results.

Funding Open Access funding provided by the IReL Consortium. This research was partially funded by Enterprise Ireland and the European Regional Development Fund (ERDF) under the National Strategic Reference Framework (NSRF) 2007-2013.

Data Availability Available on Open Science Framework, OSF: Resource 1 https://osf.io/7c2va/ MS Excel Spreadsheet of all swallow pressure-time swallow vectors. Resource 2 https://osf.io/n9gw8/ MS Excel Spreadsheet of all swallow vectors aligned on Pmax. Resource 3 https://osf.io/bf8sw/ Supplementary Figures. Resource 4 https://osf.io/ hp4bg/ Threshold Excess Swallow Analysis. Resource 5 https://osf.io/ $68 \mathrm{a} 5 \mathrm{c} /$ Python Code for swallow data graphical analysis and rendering.

\section{Declarations}

Conflict of interest The authors have no relevant financial or non-financial interests to disclose.
Ethical Approval Ethics approval for this study, which conformed to the Helsinki Declaration, was obtained from the University of Limerick, Faculty of Education and Health Sciences research ethics committee. Approval to use a novel medical device, OroPress, in this clinical investigation was approved by the designated authority for Ireland, the Health Products Regulatory Authority (HPRA) with reference numbers CI0053\#0 - CRN 2148129 EUDAMED Reference Number: CIVIE-14-06-012200, 15 Dec 2015.

Consent to Participate Participants were recruited by use of advertising posters and verbal requests. A total of 35 normal healthy adults were purposefully recruited (17 males and 18 females). Participants were screened by use of a short questionnaire about their past and present swallowing function. Exclusion criteria included having a history of a swallowing and/or speech disorder; having a medical condition, or use of medications, that may affect swallowing. An oro-motor examination excluded people with any oral abnormality and those with an overly sensitive gag reflex (i.e., gag reflex triggered in the middle portion of the anterior tongue). Persons unable to give informed consent or to follow oral instructions were also excluded. Informed consent was obtained from all individual participants included in the study.

Consent for Publication Informed consent was obtained from all individual participants included in this clinical investigation.

Open Access This article is licensed under a Creative Commons Attribution 4.0 International License, which permits use, sharing, adaptation, distribution and reproduction in any medium or format, as long as you give appropriate credit to the original author(s) and the source, provide a link to the Creative Commons licence, and indicate if changes were made. The images or other third party material in this article are included in the article's Creative Commons licence, unless indicated otherwise in a credit line to the material. If material is not included in the article's Creative Commons licence and your intended use is not permitted by statutory regulation or exceeds the permitted use, you will need to obtain permission directly from the copyright holder. To view a copy of this licence, visit http://creativecommons.org/licenses/by/4.0/.

\section{References}

1. Katakam, A., Gupta, V., Unnikrishnan, R., \& Narayanan, J. (2000). Measurements of acceleration during videofluorographic evaluation of dysphagic patients. Medical Engineering and Physics, 22(6), 405-412. https://doi.org/10.1016/S1350-4533(00) 00047-3

2. Leslie, P. (2016). Physiology of swallowing in basic clinical radiobiology (5th ed.). Taylor and Francis.

3. Farland, M.G. (2011). Under pressure: Generic and individual intra-oral pressure profiles in liquid swallows. Doctoral dissertation, University of Otago, Dunedin, New Zealand

4. Bloem, B. R., Lagaay, A. M., van Beek, W., Haan, J., Roos, R. A., \& Wintzen, A. R. (1990). Prevalence of subjective dysphagia in community residents aged over 87. BMJ : British Medical Journal, 300(6726), 721-722.

5. Youmans, S. R., Youmans, G. L., \& Stierwalt, J. A. G. (2009). Differences in tongue strength across age and gender: Is there a diminished strength reserve? Dysphagia, 24(1), 57-65. https:// doi.org/10.1007/s00455-008-9171-2

6. Utanohara, Y., Hayashi, R., Yoshikawa, M., Yoshida, M., Tsuga, K., \& Akagawa, Y. (2008). Standard values of maximum tongue pressure taken using newly developed disposable tongue pressure 
measurement device. Dysphagia, 23(3), 286-90. https://doi.org/ 10.1007/s00455-007-9142-z

7. Steele, C. M. (2013). Optimal approaches for measuring tonguepressure functional reserve. Journal of Aging Research, 2013, 1-7. https://doi.org/10.1155/2013/542909

8. Manning, M., Casey, V., Conway, R., Saunders, J., \& Perry, A. (2016). A study of healthy adults' oro-lingual effort during swallowing using oropress, a new portable wireless measurement tool. Dysphagia, 31, 442-451. https://doi.org/10.1007/ s00455-016-9697-7

9. Nomura, K., Utsumi, A., Tomita, K., Watanabe, M., Ooka, T., Hironaka, S., \& Mukai, Y. (2011). Influence of command on tongue elevation during swallowing: Exammination of tongue pressure and ultrasound imaging. Journal of Disability and Oral Health, 12(4), 149-158.

10. Matsuo, K., \& Palmer, J. B. (2008). Anatomy and physiology of feeding and swallowing: Normal and abnormal. Physical Medicine and Rehabilitation Clinics of North America, 19(4), 691-707. https://doi.org/10.1016/j.pmr.2008.06.001

11. Casey, V., Grace, P., \& Clarke-Moloney, M. (2019). Pressure measurement at biomedical interfaces, in applied biomedical engineering. InTech. Retrieved May 29, 2011 from http://www.intec hopen.com/articles/show/title/pressure-measurement-at-biome dical-interfaces

12. Kennedy, D., Kieser, J., Bolter, C., Swain, M., Singh, B., \& Waddell, J. N. (2010). Tongue pressure patterns during water swallowing. Dysphagia, 25(1), 11-19. https://doi.org/10.1007/ s00455-009-9223-2

13. Santander, P., Engelke, W., Olthoff, A., \& Volter, C. (2013). Intraoral pressure patterns during swallowing. European Archives of Oto-Rhino-Laryngology, 270(3), 1019-1025. https://doi.org/10. 1007/s00405-012-2299-6

14. Subasi, A., \& Gursoy, M. I. (2010). EEG signal classification using PCA, ICA, LDA and support vector machines. Expert Systems with Applications, 37, 8659-8666.

15. Stierwalt, J. A., \& Youmans, S. R. (2007). Tongue measures in individuals with normal and impaired swallowing. The American Journal of Speech-Language Pathology, 16(2), 148-56. https:// doi.org/10.1044/1058-0360(2007/019)

16. Nicosia, M., Hind, J., Roecker, E. B., Carnes, M., Doyle, J., Dengel, G.A., \& Robbins, J. (2000). Age effects on the temporal evolution of isometric and swallowing pressure. The Journals of Gerontology, Series A: Biological Sciences, 55, 634-640.

17. Lindgren, S., \& Janzon, L. (1991). Prevalence of swallowing complaints and clinical findings among 50-79-year-old men and women in an urban population. Dysphagia, 6(4), 187-192. https:// doi.org/10.1007/BF02493524

18. McCormack, J., Casey, V., Conway, R., Saunders, J., \& Perry, A. (2015). Oropress a new wireless tool for measuring oro-lingual pressures: A pilot study in healthy adults. Journal of NeuroEngineering and Rehabilitation, 12(1), 1-9. https://doi.org/10.1186/ s12984-015-0024-6

19. Cummings, K., Laws, P., Redish, E., \& Cooney, P. (2004). Understanding physics. Wiley.

20. Harster, P. (2005). Tissue modeling: The oral pump. Quintessence International, 36(8), 633-640.

21. Casey, V., \& OSullivan, S., \& McEwen, J. A. (2004). Interface pressure sensor for ivra and other biomedical applications. Medical Engineering and Physics, 26(2), 177-82.
22. Engelke, W., Jung, K., \& Knosel, M. (2011). Intra-oral compartment pressures: A biofunctional model and experimental measurements under different conditions of posture. Clinical Oral Investigations, 15(2), 165-176. https://doi.org/10.1007/ s00784-009-0367-0

23. Kieser, J., Bolter, C., Raniga, N., \& Waddell, N. (2010). Stability of intraoral pressure patterns during swallowing. Dysphagia, 25(4), 362-363.

24. Ono, T., Hori, K., Masuda, Y., \& Hayashi, T. (2010). Recent advances in sensing oropharyngeal swallowing function in Japan. Sensors (Basel, Switzerland), 10(1), 176-202. https://doi.org/10. 3390/s100100176

25. Ball, S., Idel, O., Cotton, S. M., \& Perry, A. (2006). Comparison of two methods for measuring tongue pressure during swallowing in people with head and neck cancer. Dysphagia, 21(1), 28-37. https://doi.org/10.1007/s00455-005-9008-1

26. Yoshikawa, M.e.a. (2021). Comparison of the iowa oral performance instrument and jms tongue pressure measurement device. Journal of Dental Sciences, 16(1), 214-219. https://doi.org/10. 1016/j.jds.2020.06.005

27. Hewitt, A., Hind, J., Kays, S., Nicosia, M., Doyle, J., Tompkins, W., Gangnon, R., \& Robbins, J. (2008). Standardized instrument for lingual pressure measurement. Dysphagia, 23(1), 16-25. https://doi.org/10.1007/s00455-007-9089-0

28. Casey, V., McAree, B., Moloney, M. C., \& Grace, P. (2002). Wearable sub-bandage pressure measurement system. In: Sensors Applications Symposium (SAS), pp. 41-45. IEEE

29. Casey, V. (2012). Biomedical interface pressure transducer for tourniquets. US Patent 20120330192.

30. Clave, P., de Kraa, M., Arreola, V., Girvent, M., Farre, R., Palomera, E., \& Serra-Prat, M. (2006). The effect of bolus viscosity on swallowing function in neurogenic dysphagia. Alimentary Pharmacology Therapeutics, 24(9), 1385-94. https://doi.org/10. 1111/j.1365-2036.2006.03118.x.

31. Alves, L. M., Cassiani Rde, A., Santos, C. M., \& Dantas, R. O. (2007). Gender effect on the clinical measurement of swallowing. Arquivos de Gastroenterologia, 44(3), 227-9.

32. van den Engel-Hoek, L., de Groot, I. J. M., Esser, E., Gorissen, B., Hendriks, J. C. M., de Swart, B. J. M., \& Geurts, A. C. H. (2012). Biomechanical events of swallowing are determined more by bolus consistency than by age or gender. Physiology and Behavior, 106(2), 285-290. https://doi.org/10.1016/j.physbeh.2012.02. 018

33. Yano, J., Kumakura, I., Hori, K., Tamine, K. I., \& Ono, T. (2012). Differences in biomechanical features of tongue pressure production between articulation and swallow. Journal of Oral Rehabilitation, 39(2), 118-125. https://doi.org/10.1111/j.1365-2842.2011. 02258.X

34. Xu, Y., Casey, V., Conway, R., \& Perry, A. (2014). PCA for tongue-palate swallowing pressure analysis. In 25th IET Irish Signals and Systems Conference 2014 and 2014 China-Ireland International Conference on Information and Communications Technologies (ISSC 2014 CIICT 2014), pp. 362-367 https://doi. org/10.1049/cp.2014.0715. IET Conference Publications.

35. WikiAudio.org: Attack, sustain, decay, release (adsr envelope). http://en.wikiaudio.org/ADSRenvelope/

36. Yoder, N. C. (2011). Peakfinder - noise tolerant fast peak finding algorithm v2.0.2.0. Mathworks.com, 26(3), 232-237. https://doi. org/10.1007/s00455-010-9291-3 